\title{
Wavefront improvement in an injection-seeded soft $x$-ray laser based on a solid-target plasma amplifier
}

\author{
Lu Li, ${ }^{1,2}$ Yong Wang, ${ }^{3}$ Shoujun Wang, ${ }^{3}$ Eduardo Oliva, ${ }^{4}$ Liang Yin, ${ }^{3}$ T. T. Thuy Le, ${ }^{4}$ Sameh Daboussi, ${ }^{2}$ David Ros, ${ }^{4}$ \\ Gilles Maynard, ${ }^{4}$ Stephane Sebban, ${ }^{2}$ Bitao Hu, ${ }^{1}$ Jorge J. Rocca, ${ }^{3}$ and Philippe Zeitoun ${ }^{2, *}$ \\ ${ }^{1}$ School of Nuclear Science and Technology, Lanzhou University, Lanzhou 730000, China \\ ${ }^{2}$ Laboratoire d'Optique Appliquee, ENSTA ParisTech, CNRS, Ecole Polytechnique, Palaiseau 91120, France \\ ${ }^{3}$ National Science Foundation Engineering Research Center for Extreme Ultraviolet Science and Technology, \\ Colorado State University, Fort Collins, Colorado 80523, USA \\ ${ }^{4}$ Laboratoire de Physique des Gaz et des Plasmas, Universite Paris XI, Orsay Cedex, France \\ ${ }^{*}$ Corresponding author: philippe.zeitoun@ensta-paristech.fr
}

Received July 30, 2013; revised September 4, 2013; accepted September 5, 2013; posted September 5, 2013 (Doc. ID 194773); published October 4, 2013

\begin{abstract}
The wavefront of an injection-seeded soft x-ray laser beam generated by amplification of high-harmonic pulses in a $\lambda=18.9 \mathrm{~nm}$ molybdenum plasma amplifier was measured by a Hartmann wavefront sensor with an accuracy of $\lambda / 32$ root mean square (rms). A significant improvement in wavefront aberrations of $0.51 \pm 0.03 \lambda \mathrm{rms}$ to $0.23 \pm 0.01 \lambda \mathrm{rms}$ was observed as a function of plasma column length. The variation of wavefront characteristic as a function time delay between the injection of the seed and peak of soft x-ray amplifier pump was studied. The measurements were used to reconstruct the soft x-ray source and confirm its high peak brightness. (C) 2013 Optical Society of America OCIS codes: (140.7240) UV, EUV, and X-ray lasers; (040.7480) X-rays, soft x-rays, extreme ultraviolet (EUV); (110.7348) Wavefront encoding.

http://dx.doi.org/10.1364/OL.38.004011
\end{abstract}

Since the first significant amplification of a soft x-ray laser (SXRL) was demonstrated in 1985 [1,ㄹ], laser driven SXRL sources have achieved remarkable progress. Their size has been greatly reduced and repetition rate has been increased by several orders of magnitude, recently reaching $100 \mathrm{~Hz}$ [3] in a table-top. These advances are opening a wealth of applications in the fields of high resolution imaging, error-free nanopatterning, photochemistry, materials characterization, and the development of nanoprobes [4]. Many of the applications are dependent on the beam quality and coherence factor, while most of the SXRLs are limited by the fact that the amplifiers are self-seed by amplified spontaneous emission (ASE). A breakthrough on the beam quality and coherence of SXRL results from injection seeding of plasma amplifiers by high harmonic generation (HHG) [5-7]. The dramatic improvement in both spatial and temporal coherence has been demonstrated to result in fully coherent beams from solid-target-based [8] and gas-based SXRL plasma amplifiers [9]. Compared to the gas-based plasma amplifier, SXRL with a solid-plasma amplifier has the advantage of producing a significantly shorter pulse due to the increased bandwidth [10], and the store of more energy per unit volume. On this basis, recently a concept of an x-ray laser based on the principle of chirped pulse amplification (CPA) that could produce high energy pulses of $100 \mathrm{~s}$ fs pulse duration has been proposed [11]. In this Letter, we concentrate on the wavefront properties of HHG-injection-seeded SXRL (SSXRL) with a solidplasma amplifier. SXRL wavefront measurements were developed by changing plasma length and time delay between the HHG seed and short pump pulse. Significant improvement of the SXRL wavefront after the plasma amplifier was observed and retropropagation was used to reconstruct the electromagnetic field near the source location.

The experiment was carried out at Colorado State University for measuring the wavefront of the HHG seed and SSXRL at $\lambda=18.9 \mathrm{~nm}, 4 d^{1} S_{0} \rightarrow 4 p^{1} P_{1}$ line of nickellike Mo, by using a Hartmann wavefront sensor developed at LOA in collaboration with Imagine Optics and SOLEIL [12]. As represented in Fig. 1, a sequence of three laser pulses from the table-top Ti:Sapphire laser $(810 \mathrm{~nm})$ were used to heat the plasma, and an additional pulse split from the same laser was used to generate the HHG seed $[8,10]$. The plasma amplifier consists of an elongated plasma $30 \mu \mathrm{m}$ wide and up to $4 \mathrm{~mm}$ in length generated by irradiation of a polished molybdenum slab target with the three pulses. An early precursor pulse $(30 \mathrm{~mJ}, 200 \mathrm{ps}$ duration) and a prepulse (250 mJ, $200 \mathrm{ps}$ duration) separated by a 5 ns delay were focused into a line, by the combination of a spherical lens and a cylindrical lens, to create a plasma approaching the Ni-like ionization stage. The plasma was allowed to expand, and was

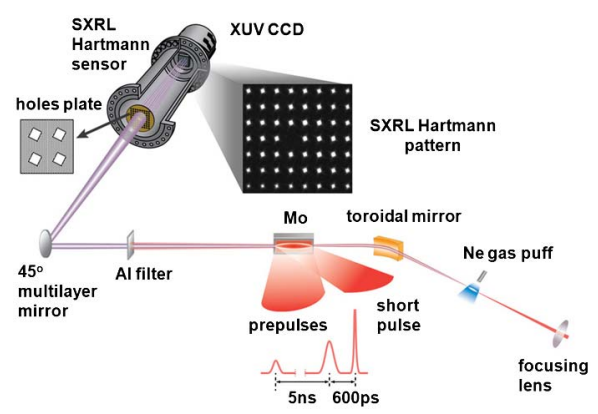

Fig. 1. Schematic diagram of the experimental setup to measure the wavefront of an injection-seeded SXRL based on solid target plasma with a Hartmann sensor. 
subsequently heated by a $500 \mathrm{~mJ}$ energy pulse with $6 \mathrm{ps}$ duration arriving at $600 \mathrm{ps}$ after the prepulse, which is focused onto a similar line as prepulses. The short heating pulse was coupled into the plasma at a grazing incident angle of $23^{\circ}$, to rapidly heat the plasma region where the electron density is $\sim 2.6 \times 10^{20} \mathrm{~cm}^{-3}$ [8]. The HHG seed pulses were generated by focusing $18 \mathrm{~mJ}$, $\sim 60$ fs duration pulses with a $1.1 \mathrm{~m}$ focal length lens into a neon gas jet (backing pressure $\sim 1.5$ bar). A toroidal mirror with $0.25 \mathrm{~m}$ focal length was used to image the harmonic source into the gain region of the amplifier. The HHG seed was injected in the plasma at a grazing angle of $5 \mathrm{mrad}$. The wavefront was diagnosed with a Hartmann wavefront sensor with an accuracy of $\lambda / 32$ root mean square (rms) at $18.9 \mathrm{~nm}$ [12]. The sensor comprises a $100 \mu \mathrm{m}$ thick nickel plate placed at about $310 \mathrm{~mm}$ in front of back-illuminated x-ray CCD. The plate contains an array of $51 \times 51$ square holes $80 \mu \mathrm{m}$ in size, separated by $380 \mu \mathrm{m}$ over a $19 \mathrm{~mm} \times 19 \mathrm{~mm}$ area. The holes are rotated by $25^{\circ}$ to prevent interference of adjacent holes. A $300 \mathrm{~nm}$ thick Al filter and $45^{\circ}$ incidence angle Mo-Si multilayer mirror designed for maximum reflectivity at $18.9 \mathrm{~nm}$ were used to reject the straight pump laser, hard $\mathrm{x}$ rays, and other spurious plasma emission. The wavefront of the SSXRL was measured by using the $45^{\circ}$ mirror and placing the sensor at $3.5 \mathrm{~m}$ away from the plasma amplifier. While the HHG seed wavefront was detected without the $45^{\circ}$ mirror, and the sensor was placed at $1.6 \mathrm{~m}$ away. As estimated, the SSXRL beam size at the $45^{\circ}$ flat mirror is $\sim 1 \mathrm{~mm}$, thus the wavefront aberration from this mirror could be neglected.

The wavefronts of both the HHG seed and SSXRL from a 2 ps delay between the HHG seed and short pump pulse were measured for several consecutive shots and compared. All measured wavefronts contain strong horizontal and vertical tilts and spherical aberration that have no impact on imaging or focusing experiments. Therefore, we only show residual wavefront distortions, in which the tilts and spherical component have been removed. The measured HHG seed wavefront distortion of $0.51 \pm 0.03 \lambda \mathrm{rms}$ is impacted by both the optical quality of the toroidal mirror and its alignment quality [Fig. 2(a)]. We also observed that the positioning and beam shape of HHG pulses are very stable from shot to shot. Figure 2(b), displays a typical 2D image of the SSXRL wavefront. It is observed that the wavefront distortion is improved to $0.22 \lambda \mathrm{rms}$ by the amplifier. However, the SSXRL beam positioning and shape are less stable from shot to shot, which depends on the plasma evolution.

We have studied the influence of the plasma column length on the SSXRL wavefront [Fig. 2(d)]. At $3 \mathrm{~mm}$ length, the wavefront remains close to $\overline{0.51 \lambda} \mathrm{rms}$, while for 3.5 and $4 \mathrm{~mm}$ the wavefront improves to reach $0.31 \lambda \mathrm{rms}$ and $0.23 \lambda \mathrm{rms}$, respectively. Since $4 \mathrm{~mm}$ long plasma presents the strongest amplification factor []] and the best wavefront, we concentrated our study on this condition. Acquiring 27 consecutive shots, we observed a wavefront stability of $0.01 \lambda \mathrm{rms}$, which is at the limit of the sensor accuracy $(\lambda / 32 \mathrm{rms})$ and three times more stable than for a direct unamplified HHG pulse. An improvement in wavefront stability with respect to the seed was also observed for 3 and $3.5 \mathrm{~mm}$ long plasmas.
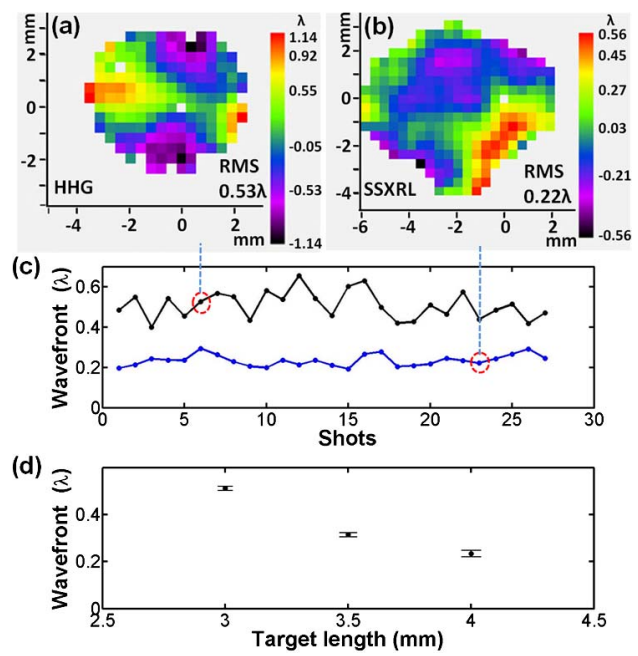

Fig. 2. Two-dimensional maps of the wavefront after removal of tilt and spherical aberrations for (a) HHG seed and (b) SSXRL. (c) Residual wavefront values of HHG seed (black line) and SXRL (4 mm target). (d) Residual wavefront error values for SSXRL from plasma lengths of 3.0, 3.5, and $4.0 \mathrm{~mm}$.

The observed beam improvement is similar to previous work that shows a strong improvement in the wavefront of SSXRL in a gas-amplifier [9]. However, the geometries of the two experiments and the physical processes influencing the gain creation are different. Consequently, the gain zone shapes are different between gas (circular gain zone with very sharp edges) and solid amplifiers (elliptical gain zone with smooth edges) $[\underline{8}, 13]$. The coupling between the seed and gain zone cannot follow the same optimization procedure and thus merits a specific study.

The wavefront sensor allows for independent measurement of the different aberrations [12]. In Fig. 3, we plotted the three strongest aberrations over 25 shots. The strongest improvement is observed for coma with values improved by more than a factor of 2 , from $0.41 \lambda$ rms to $0.18 \lambda \mathrm{rms}$. Coma generates a central spot surrounded by many rays extending far from it [14]. This spot is selectively amplified while the surrounding rays are much less amplified, generating the observed drop of coma aberration, which is the so-called spatial filtering effect. Astigmatism at $0^{\circ}$ drops from $\sim 0.10 \lambda$ rms to the detection limit ( $0.03 \lambda \mathrm{rms})$, while astigmatism at $45^{\circ}$ does not show significant changes.
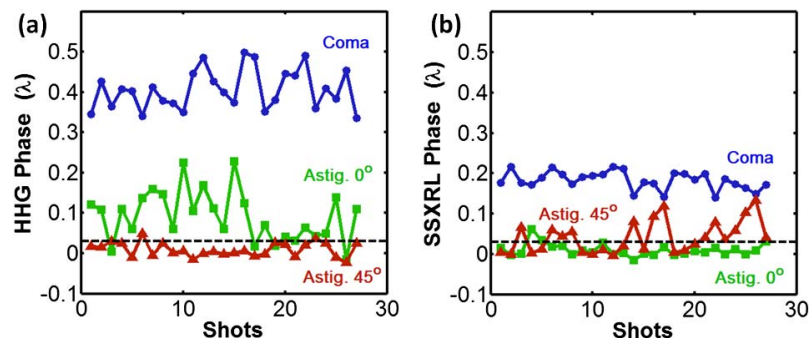

Fig. 3. Shot-to-shot evolution of the three main aberrations, coma (blue line), astigmatism at $0^{\circ}$ (green line), and astigmatism at $45^{\circ}$ (red line) for both (a) HHG seed and (b) SSXRL with a $4 \mathrm{~mm}$ long plasma amplifier. The detection limit of the Hartmann wavefront sensor is indicated by the black dashed line. 


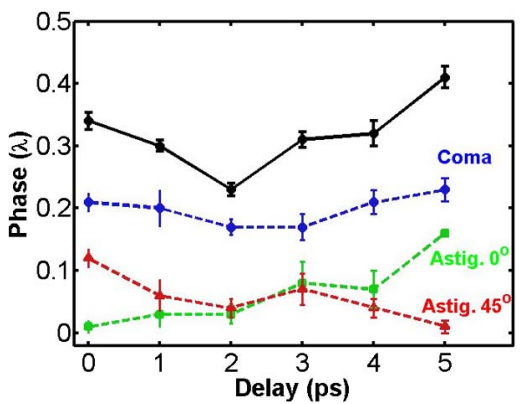

Fig. 4. Total measured SSXRL wavefront distortion (black line, top curve). Aberrations as a function of delay between the injection of the HHG seed and the peak of the amplifier pump pulse for coma (blue dashed line), astigmatism at $0^{\circ}$ (greendashed line), and astigmatism at $45^{\circ}$ (red-dashed line).

Solid amplifiers being pumped by a combination of long and short pulses are known to experience a hydrodynamic evolution that may impact the wavefront of the amplified HHG seed. To study this effect, the HHG seed was injected at different delays ranging from $0 \mathrm{ps}$ (seed synchronized with the peak of a short pump pulse) to +5 ps delay (Fig. 4).We observed $\sim 250$ times and $\sim 400$ times amplification with the $\sim 1 \mathrm{~nJ}$ HHG seed at the delay of 2 and 3 ps, respectively. But after the 5 ps delay, the intensity of the amplified signal is too weak to enable single-shot wavefront detection with the Hartmann wavefront sensor. Therefore, we conducted measurements for a span of 5 ps for a $4 \mathrm{~mm}$ long plasma amplifier. An optimum wavefront of $0.23 \lambda \mathrm{rms}$ was observed at 2 ps delay, while the wavefront aberration increases up to $0.41 \lambda \mathrm{rms}$ at longer delays. Astigmatism at $45^{\circ}$ decreased from $0.12 \lambda$ rms at 0 ps to the detection limit at $5 \mathrm{ps}$, and astigmatism at $0^{\circ}$ was observed to increase with the delay, while coma aberration showed very little variation with delay. In a different set of measurements the incident angle of the HHG seed was changed to $10 \mathrm{mrad}$ with no clear effect on the wavefront. Although these trends are associated with the evolution of plasma parameters such as density gradients, size, shape, and position of the gain region, a completely understanding of these results requires coupling 3D Maxwell-Bloch codes with 2D or 3D hydrodynamic codes. Currently, to our best knowledge, such a model does not exist. While we are progressing along this direction, the current work is limited to the above observations.

To further understand the impact of the hydrodynamic conditions on the SSXRL wavefront, we took the benefit of the capacity of the wavefront sensor to also simultaneously record the intensity map. Thus, the complex electromagnetic field may be reconstructed at the Hartmann plate of the sensor and the SSXRL wave field may be calculated at any location by an inverse Fresnel transformation. Such reconstruction is done by using the Imagine Optic software that has been tested against a model with excellent agreement [15]. The main issue during retropropagation is the choice of the pupil size. Large pupils contain noise from plasma ASE that has to be removed. Conversely, reducing too much of the pupil generates incorrect intensity distribution and spatial resolution, and also results in smoothing of the outer part of the beam where aberrations imprint most of the wavefront defects. We thus selected the largest pupils so as to contain about $95 \%$ of the incoming energy and still remove most of the noise. The second issue when reconstructing the intensity distribution near the source location is the spatial resolution along both the transversal and longitudinal axis. As given by the numerical aperture of the beam, the transverse resolution is about $10 \mu \mathrm{m}$ while the Rayleigh length is about $20 \mathrm{~mm}$. Consequently, we were not able to resolve the fine structure of the gain zone. However, it was possible to follow the change of the source size and shape for the different time delays. We calculated the transverse intensity distribution at different positions along the propagation direction $(Z=-20,0$, and $20 \mathrm{~mm}$ ) of the plasma along the propagation direction [Figs. 5(a) and 5(b)]. For $Z=-20$ and $20 \mathrm{~mm}$, astigmatism produces elongated beams, FWHM of $14 \mu \mathrm{m} \times 10 \mu \mathrm{m}$ and $13 \mu \mathrm{m} \times 11 \mu \mathrm{m}$, respectively, and with main axis rotated by about $45^{\circ}$. The source itself $(Z=0 \mathrm{~mm})$ is relatively circular with a spot size of $11 \mu \mathrm{m} \times 12 \mu \mathrm{m}$ FWHM. Although the transverse intensity distribution was calculated at several locations, here we displayed only the most interesting case corresponding to the gain region. Figure 5(c) shows the source profile for three different delays between the HHG seed and the peak of the short pump pulse. At 2 ps delay, the source zone is the smallest $(12 \mu \mathrm{m} \times 14 \mu \mathrm{m}$ FWHM $)$ which is consistent with the time of the best wavefront in Fig. 4 . Also, the 0 ps case is consistent with Fig. 4 showing a slightly elongated source with an angle of about $45^{\circ}$ with respect to the vertical axis that might be given by the astigmatism in that direction. At a delay of $4 \mathrm{ps}$, the source is roughly circular agreeing with weak astigmatism $45^{\circ}$.

In summary, we have measured the wavefront of an SSXRL created by irradiation of a solid target by using a soft $\mathrm{x}$ ray Hartmann wavefront sensor. The main conclusion of this experiment is that a SXRL solid-based plasma amplifier (4 mm long) produces a good wavefront

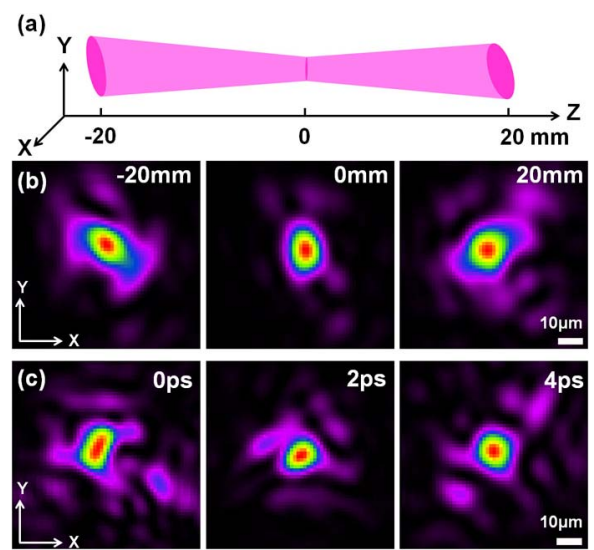

Fig. 5. (a) Diagram of reconstructed SSXRL propagation around the plasma amplifier along the Rayleigh length. (b) False color images of the reconstructed SSXRL transverse intensity distribution at $Z=-20,0$, and $20 \mathrm{~mm}$, with $Z=0 \mathrm{~mm}$ corresponding to the central position of the plasma and also to the region of least confusion. The delay is at $2 \mathrm{ps}$. (c) Reconstructed SSXRL intensity maps at $Z=0 \mathrm{~mm}$ for various delays between HHG seed and pump laser pulse. The length of the plasma amplifier is $4 \mathrm{~mm}$. The case at $0 \mathrm{ps}$ corresponds to the seed arriving at the peak of the amplifier short pump pulse. 
with distortions reaching $\lambda / 5 \mathrm{rms}$, although it is significantly denser than a gas-based amplifier. Previous work on a gas-plasma amplifier [9] shows that the small size of gain region acts as a spatial filter of the incoming beam and results in a reduction of the wavefront aberration for the amplified signal. In this experiment, we found that the wavefront improvement factor is $\sim 2.5$, and the main optimization is coma aberrations by this effect. Second, the optimized wavefront occurs at a 2 ps delay between HHG seed and the peak of the 6 ps pump pulse and the more sensitive distortion is astigmatism, while coma is relatively stable by the delay. We also reconstructed the beam intensity distribution near the plasma location under different time delays. To perform a more detailed study of the impact of plasma hydrodynamics, an image relay system must be coupled to the wavefront sensor, or a small pinhole must be placed in the beam path to achieve a higher numerical aperture and higher spatial resolution. Moreover, the statistical studies observed that the wavefront of the SSXRL beam is more stable than that of the HHG seed, opening the possibility to implement static or active wavefront correction. Finally, this measurement of the source size of a seeded solid target SXRL confirms the high brightness of $\sim 1 \times$ $10^{26}$ photons $\cdot \mathrm{s}^{-1} \cdot \mathrm{mm}^{-2} \cdot \mathrm{mrad}^{-2}$. As a comparison, the SXRL peak brillance from a free-electron laser in FLASH is $1 \times 10^{29}-1 \times 10^{31}$ photons $\cdot \mathrm{s}^{-1} \cdot \mathrm{mm}^{-2} \cdot \mathrm{mrad}^{-2} / 0.1 \% \mathrm{BW}$.

The authors acknowledge assistance from Imagine Optics, and thank Eric Gullickson for the fabrication of the multilayer mirror. This work was supported by SHYLAX, RTRA "Triangle de la Physique," INREX in LASERLAB3 and FP7 European program n ${ }^{\circ} 284464$, and by the AMOS program of the Office of Basic Energy Science, US Department of Energy, and by the NSF ERC for Extreme Ultraviolet Science and Technology with equipment development under NSF Award MRI-ARRA 09-561.

\section{References}

1. D. Matthews, P. Hagelstein, M. Rosen, M. Eckart, N. Ceglio, A. Hazi, H. Medecki, B. MacGowan, J. Trebes, B. Whitten, E. Campbell, C. Hatcher, A. Hawryluk, R. Kauffman,
L. Pleasance, G. Rambach, J. Scofield, G. Stone, and T. Weaver, Phys. Rev. Lett. 54, 110 (1985).

2. S. Suckewer, C. H. Skinner, H. Milchberg, C. Keane, and D. Voorhees, Phys. Rev. Lett. 55, 1753 (1985).

3. B. A. Reagan, K. A. Wernsing, A. H. Curtis, F. J. Furch, B. M. Luther, D. Patel, C. S. Menoni, and J. J. Rocca, Opt. Lett. 37, 3624 (2012).

4. L. Urbanski, A. Isoyan, A. Stein, J. J. Rocca, C. S. Menoni, and M. C. Marconi, Opt. Lett. 37, 3633 (2012).

5. T. Ditmire, M. Hutchinson, M. Key, C. Lewis, A. MacPhee, I. Mercer, D. Neely, M. Perry, R. Smith, J. Wark, and M. Zepf, Phys. Rev. A 51, R4337 (1995).

6. P. Zeitoun, G. Faivre, S. Sebban, T. Mocek, A. Hallou, M. Fajardo, D. Aubert, P. Balcou, F. Burgy, D. Douillet, S. Kazamias, G. De Lacheze-Murel, T. Lefrou, S. Le Pape, P. Mercere, H. Merdji, A. S. Morlens, J. P. Rousseau, and C. Valentin, Nature 431, 426 (2004).

7. Y. Wang, E. Granados, M. A. Larotonda, M. Berrill, B. M. Luther, D. Patel, C. S. Menoni, and J. J. Rocca, Phys. Rev. Lett. 97, 123901 (2006).

8. Y. Wang, E. Granados, F. Pedaci, D. Alessi, B. Luther, M. Berrill, and J. J. Rocca, Nat. Photonics 2, 94 (2008).

9. J. P. Goddet, S. Sebban, J. Gautier, P. Zeitoun, C. Valentin, F. Tissandier, T. Marchenko, G. Lambert, M. Ribieres, D. Douillet, T. Lefrou, G. Iaquaniello, F. Burgy, G. Maynard, B. Cros, B. Robillard, T. Mocek, J. Nejdl, M. Kozlova, and K. Jakubczak, Opt. Lett. 34, 2438 (2009).

10. Y. Wang, M. Berrill, F. Pedaci, M. M. Shakya, S. Gilbertson, Z. Chang, E. Granados, B. M. Luther, M. A. Larotonda, and J. J. Rocca, Phys. Rev. A 79, 023810 (2009).

11. E. Oliva, M. Fajardo, L. Li, M. Pittman, T. T. T. Le, J. Gautier, G. Lambert, P. Velarde, D. Ros, S. Sebban, and P. Zeitoun, Nat. Photonics 6, 764 (2012).

12. P. Mercere, Ph. Zeitoun, M. Idir, S. L. Pape, D. Douillet, X. Levecq, G. Dovillaire, S. Bucourt, K. A. Goldberg, P. P. Naulleau, and S. Rekawa, Opt. Lett. 28, 1534 (2003).

13. M. Berrill, D. Alessi, Y. Wang, S. R. Domingue, D. H. Martz, B. M. Luther, Y. Liu, and J. J. Rocca, Opt. Lett. 35, 2317 (2010).

14. M. Born and E. Wolf, Principles of Optics (Cambridge University, 1999), pp. 237-240.

15. G. Lambert, F. Tissandier, J. Gautier, C. P. Hauri, P. Zeitoun, C. Valentin, T. Marchenko, J. P. Goddet, M. Ribiere, A. Sardinha, M. Fajardo, F. Hamouda, G. Maynard, G. Rey, and S. Sebban, EPL 89, 24001 (2010). 